\title{
Erratum zu: Kritische Anmerkungen zur Vermeidung des Erziehungsbegriffs
}

\author{
Dominik Krinninger
}

\section{Erratum zu:}

Kapitel „Kritische Anmerkungen zur Vermeidung des Erziehungsbegriffs" in: W. Meseth et al. (Hrsg.), Normativität in der Erziehungswissenschaft, https://doi.org/10.1007/978-3-658-21244-5_13

Im Abschnitt 3 „Erziehung als peripheres Thema der Erziehungswissenschaft oder: Auf wen sich Erziehung richtet, bleibt unterbestimmt" wurde anstelle von

„Klaus Prange entwirft in seiner Untersuchung über Die Zeigestruktur der Erziehung (Prange 2005), Erziehung als Zeigen im Dreieck zwischen Kind, Erzieher und Thema bzw. Sache, wobei der jeweilige Status von bzw. die Differenzen zwischen Kind und Erzieher kaum thematisiert werden."

irrtümlich geschrieben

„Klaus Prange entwirft in seiner Untersuchung über Die Zeigestruktur der Erziehung (Prange 2005), Erziehung als statt: über die „Zeigestruktur der Erziehung“, die er als Grundriss der operativen Pädagogik (2005) vorlegt, Erziehung als Zeigen im Dreieck zwischen Kind, Erzieher und Thema bzw. Sache, wobei der jeweilige Status von bzw. die Differenzen zwischen Kind und Erzieher kaum thematisiert werden.“

Dies wurde jetzt korrigiert.

Die korrigierte Version des Kapitels ist verfügbar unter https://doi.org/10.1007/978-3-658-21244-5_13 